\title{
Nivel de conocimiento de defectos de esmalte y su tratamiento entre odontopediatras.
}

María Gabriela Acosta de Camargo";

Alfredo Natera ${ }^{2}$;

\section{Resumen}

Objetivo: Evaluar el conocimiento de defectos de esmalte entre odontólogos especialistas en Odontopediatría y tratamientos disponibles Material y método: Fue una investigación de tipo descriptiva con un diseño de campo no experimental, con encuesta transversal. La población objeto de estudio estuvo conformada por 31 especialistas de Odontopediatría, practicantes en Venezuela, quienes provenían de 7 universidades de diferentes países. Resultados: Se encontró que $77,41 \%$ de los encuestados sabían el defecto de esmalte que estaban observando, específicamente fluorosis, y 51,61\% Pigmentación. De los encuestados $41.93 \%$ acertaron con el diagnóstico de Hipomineralización Molar Incisivo. Los tratamientos más conocidos entre odontopediatras fueron la microabrasión (90.32\%), blanqueamiento $(58.06 \%)$, carillas $(54.83 \%)$ y resinas (51.61\%). Conclusión: Los odontopediatras consultados en el presente estudio mostraron un nivel aceptable de conocimiento hacia los defectos de esmalte y los tratamientos disponibles para mejorar la apariencia de los mismos.

Palabras clave: Conocimiento, defectos de esmalte, odontopediatras

Artigo Original

\section{Nível de conhecimento dos defeitos de esmalte e tratamento entre os odontopediatras}

\section{Resumo}

Alvo: Avaliar o conhecimento dos defeitos do esmalte dentário entre os especialistas em odontologia pediátrica e tratamentos disponíveis para o tratamento. Material e método: Foi um projeto de pesquisa descritiva, com um campo não-experimental, com estudo transversal. A população do estudo consistiu de 31 especialistas em odontopediatria, praticantes na Venezuela, que vieram de sete universidades em diferentes países. Resultados: Verificou-se que $77,41 \%$ dos entrevistados sabiam foram observados os defeitos do esmalte, especificamente fluorose, e 51,61\% pigmentação. $41,93 \%$ dos entrevistados combinado com o diagnóstico de hipomineralização incisivo Molar. O

\footnotetext{
${ }^{1}$ Odontopediatra. Profesora asociada del Departamento de Odontología del Nino y del Adolescente. Universidad de Carabobo. Venezuela.

${ }^{2}$ Profesor de la Cátedra de Odontología Operatoria. Universidad Central de Venezuela.
} 
mais conhecido entre os dentistas tratamentos pediátricos foram Microabrasão (90,32\%), branquear $(58,06 \%)$, folheados $(54,83 \%)$ e resinas $(51,61 \%)$. Conclusão odontopediatras pesquisados neste estudo mostraram um nível aceitável de conheci- mento ao esmalte defeitos e tratamentos disponíveis para melhorar a aparência dos mesmos.

Palavras-chave: conhecimento, defeitos de esmalte, dentistas pediátricos

Review Article

\section{Level of knowledge concerning enamel defects and their treatment among pediatric dentists}

\section{Abstract}

Objective: Assess the level of knowledge of dental enamel defects among specialists in pediatric dentistry and available treatment. Material and method: It was a descriptive research design with a non-experimental field, with cross-sectional survey. The study population consisted of 31 pediatric dentistry specialists, practitioners in Venezuela, who came from seven universities in different countries. Results: It was found that $77.41 \%$ of respondents knew the enamel defects were observed, specifically fluorosis, and $51.61 \%$ pigmentation, $41.93 \%$ of respondents matched with the diagnosis of hypomineralization Molar incisor. The best known treatments among pediatric dentists were Microabrasion (90.32\%), bleaching $(58.06 \%)$, veneers $(54.83 \%)$ and resins (51.61\%). Conclusion: Pediatric dentists surveyed in this study showed an acceptable level of knowledge to enamel defects and treatments available to improve the appearance thereof.

Keywords: Knowledge, enamel defects, pediatric dentists

\section{Introducción}

Los defectos de esmalte (DDE) son un conjunto de alteraciones clínicamente visibles en el esmalte, debidos a desórdenes ocurridos durante la biomineralización o en la secreción de la matriz del esmalte. ${ }^{1}$ Cualquier disturbio durante la formación del esmalte genera cambios permanentes, ya que el ameloblasto, célula formadora del esmalte, de origen ectodérmico y altamente especializada, tiene escasa capacidad reparativa. ${ }^{2}$

La importancia del diagnóstico de DDE se ha asociado al aumento de presencia de caries, fracturas dentales, sensibilidad, manejo de la conducta en el consultorio, alteraciones psicológicas, problemas estéticos ${ }^{3-5}$ entre otros.

Los DDE representan problemas comunes dentro de la población y favorecen la formación de caries dental, tanto en la dentición primaria como en la permanente..$^{6-8} \mathrm{El}$ profesional de la odontología debe conocer los factores de riesgo y ofrecer tratamientos conservadores, estéticos y protésicos que puedan brindarle al paciente una odontología cosmética que disminuya la visualización de estos defectos y le ayude al paciente a tener soluciones efectivas de un problema ampliamente 
estudiado. En muchos casos su diagnóstico no es preciso y por falta de conocimiento no se brinda una atención temprana adecuada. Los DDE poseen una variedad de manifestaciones clínicas, ya que dependiendo del momento en que ocurren durante la formación dentaria, será la clínica que se observará. Es importante conocer su etiopatogenia, saber cómo se producen las alteraciones, diferenciarlas y ofrecer alternativas que ayuden a disminuir su evidencia, favoreciendo tanto la estética como la función. ${ }^{9}$

La prevalencia de los DDE varía según la población que se estudie. Investigaciones realizadas en Brasil $^{7}$, Malaysia ${ }^{10}$ y Tonga ${ }^{11}$ reportan una prevalencia de DDE del 29,7\% sin fluorosis dental. ${ }^{7} \mathrm{Sin}$ embargo, en China ${ }^{12}$ y Nueva Zelandia ${ }^{13}$ se registran niveles de hasta $100 \%$. La variación de datos se debe a la tipos de defectos estudiados; diferentes clasificaciones de los índices o los índices con modificación establecida; diferentes configuraciones de campo y los procedimientos de examen técnico, como la iluminación o si los dientes se secaron o no; y factores en la población, como status socioeconómico, factores genéticos, raciales y étnicos. ${ }^{7,13}$

Los factores etiológicos de DDE han sido relacionados con: bajo peso al nacer, ${ }^{14}$ enfermedades sistémicas como asma, ${ }^{15}$ enfermedad celía$\mathrm{ca}^{16}{ }^{16}$ malnutrición, ${ }^{17}$ enfermedades renales, ${ }^{18-21}$ virus de varicela, exposición a cigarrillo, otitis, consumo de pastas dentales, ${ }^{22}$ índice de masa corporal. ${ }^{5}$

Los sujetos que cursan con DDE muestran aislamiento social y angustia, elevada disfunción, malestar y discapacidad, atribuible a su condición bucal, entendiéndose que los defectos de esmalte tiene un marcado impacto en la salud psicosocial de personas afectadas, especialmen- te a edades tempranas. ${ }^{23}$ Así como también ocasionan efectos negativos en la percepción de la salud bucal del niño y en su desempeño diario. ${ }^{24}$ En el Reino Unido se llevó a cabo un estudio en pacientes entre 7-16 años, con defectos en el esmalte visibles de los incisivos permanentes y que habían recibido microabrasión, con o sin restauración de resina adicional. Antes del tratamiento, los niños reportaron altos niveles de preocupación, la vergüenza y la percepción de que sus dientes parecían amarillos y descoloridos. Después del tratamiento, pensaban que sus dientes se veían mucho mejor, se sentían más felices y con más confianza. ${ }^{25}$

Desde 1901 se hizo la primera identificación de un defecto de esmalte por McKay. ${ }^{8}$ Se han ideado numerosos índices con el fin de identificar y clasificar los DDE, éstos se han divido en dos grupos principalmente, unos que describen la fluorosis y otro los defectos de esmalte. El más usado en fluorosis es el de Dean ${ }^{26}$ y en el resto de los defectos, unos de los que ha sido ampliamente usados, han sido el índice de Defectos del Desarrollo de esmalte, modificado por Clarkson y $\mathrm{O}^{\prime} \mathrm{Mullane}^{27}$, así como el de $\mathrm{FDI}^{28}$. Sin embargo, al estudiar detenidamente la mayoría de índices se sabe que existen numerosos vacíos. ${ }^{1}$

Con el desarrollo de criterios de juicio de la Academia Europea de Odontología Pediátrica (EAPD), ha habido un creciente interés en todo el mundo en la investigación de la prevalencia de opacidades demarcadas de sustancia de esmalte de los dientes, conocidos como Hipomineralización Molar Incisivo (MIH). Sin embargo, la falta de un sistema estandarizado con el fin de registrar los datos de MIH en encuestas epidemiológicas ha contribuido en gran medida a las amplias variaciones en la prevalencia entre estudios. Se requiere de validación del método de clasifica- 
ción, y su fiabilidad y utilidad, así como es necesario ser probado en diferentes grupos de edad y diferentes poblaciones. ${ }^{29}$

Tratamientos disponibles para defectos de esmalte.

Actualmente existen numerosos tratamientos estéticos y cosméticos para mejorar o disimular la apariencia de los DDE. Entre ellos se destacan: la microabrasión, los blanqueamientos, la macroabrasión, uso de carillas y porcelanas.

La microabrasión es una técnica conservativa, ideal para eliminar manchas superficiales de esmalte. Además puede completarse con blanqueamiento para mejores resultados. ${ }^{30,31}$

Considerando la alta incidencia de niños que presentan manchas hipoplásicas o fluorosis en dientes permantentes, se han comparado técnicas de microabrasión en odontopediatria para evaluar efectividad, encontrando que el uso de ácido hipoclorhídrico al 10\% con piedra pómez muestra buenos resultados. Aunque también se ha sustituido este último por ácido fosfórico al $37 \%$ asociado también a piedra pómez en proporción 1:1. ${ }^{30}$ Igualmente se ha indicado el uso de ácido hipoclorhídrico al 6\% con sílica aplicado con una presión mecánica con copa de goma a baja velocidad ${ }^{32}$. Estudios clínicos apoyan la eficacia y longevidad de este tratamiento seguro y mínimamente invasivo ${ }^{33,34}$, obteniendo un resultado muy satisfactorio, logrando la eliminación total de las pigmentaciones producto de la fluorosis y mejorando notablemente la superficie del esmalte mediante un tratamiento conservador y permanente. ${ }^{35}$

Existe también la macroabrasión, técnica usada para la remoción de manchas superficiales lo- calizadas que pueden mejorar notablemente la apariencia $^{36}$ en la cual se hace un desgaste mayor a través de sistemas rotatorios de alta velocidad.

En los tratamientos para DDE, se ha propuesto el uso también de carillas de porcelana, las cuales deben ser aplicadas después de realizar una evaluación multidisciplinaria para evaluar si realmente su aplicación es necesaria. ${ }^{37}$

Es imperativo conocer los defectos de esmalte e identificarlos para poder explicar incluso a los padres lo que ocurre en la boca del paciente. Sin embargo, se hace necesario saber si como especialistas se recibe la suficiente capacitación para manejar términos y establecer diferencias entre la variedad de DDE que existe. Antes de ofrecer tratamientos alternativos, cosméticos y estéticos, el conocimiento científico debería prevalecer.

Esta investigación tuvo como objetivo evaluar el conocimiento de identificación defectos de esmalte entre odontólogos especialistas en Odontopediatría y tratamientos disponibles.

\section{Material y Método.}

Es una investigación de tipo descriptiva con un diseño de campo no experimental, con encuesta transversal. La población objeto de estudio, para la presente investigación estuvo conformada por especialistas de Odontopediatría, practicantes en Venezuela, quienes provenían de 7 diferentes universidades de varios países. Universidad Central de Venezuela, Universidad de Carabobo, Universidad del Zulia, Universidad Santa María, Universidad Tecnológica de México, Universidad Javeriana, Universidad de Nueva York. 


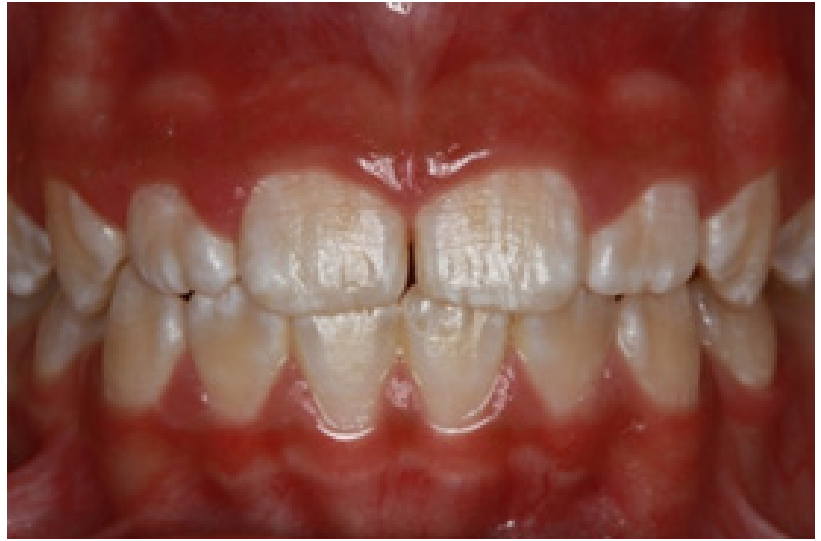

Figura 1. Fluorosis dental.

La investigación fue desarrollada mediante el uso de una encuesta estructurada, enviada a especialistas de Odontopediatría. La encuesta fue enviada a 40 odontopediatras de los cuales 31 contestaron. La muestra estuvo conformada por 31 odontopediatras quienes recibieron una encuesta vía correo electrónico. La encuesta estuvo estructura en 3 imágenes y una pregunta, cuyo tiempo de respuesta tomaba entre 3-5 minutos. Fue una sola pregunta por imagen, tipo cerrada.

Las imágenes fueron de pacientes que dieron su consentimiento informado para que la encuesta se desarrollara (Figura 1, Figura 2 y Figura 3).

El procedimiento para determinar la validez del instrumento se realizó mediante el juicio de

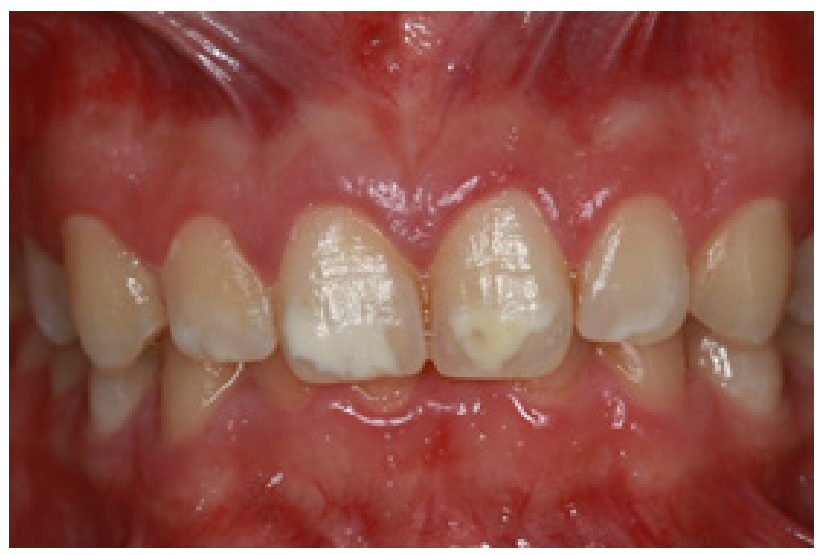

Figura 2. Opacidad demarcada.

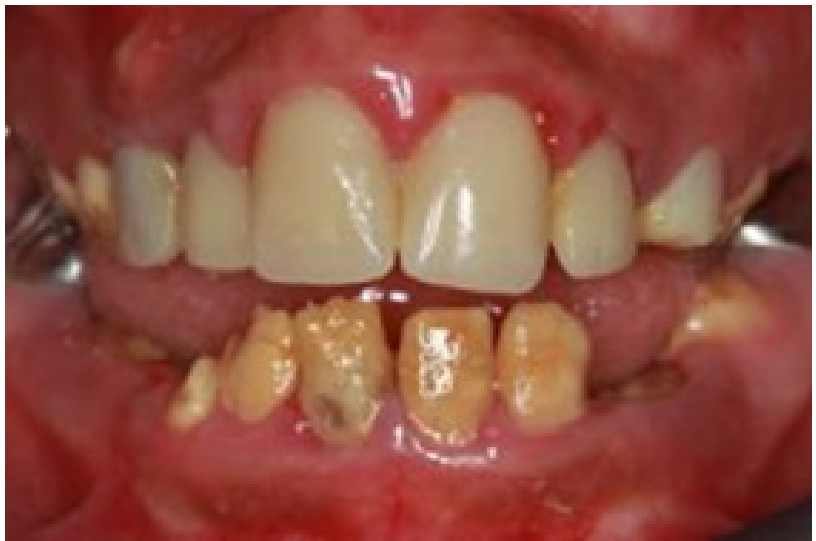

Figura 3. Amelogénesis imperfecta.

un número impar de expertos en las Áreas de Odontopediatría (2) y en el Área de Metodología de la Investigación (1). Los datos obtenidos a través de la encuesta fueron analizados y procesados utilizando técnica de estadística descriptiva mediante el programa SPSS.

\section{Resultados}

En la Tabla No. 1 puede observarse que después de que los encuestados observaran la primera imagen de la encuesta (Figura 1), para evaluar el conocimiento que tenían los odontopediatras de DDE, se encontró que 77,41\% de los encuestados sabían el defecto de esmalte que estaban observando, específicamente fluorosis. Hubo un

\begin{tabular}{|l|c|c|}
\hline & F. A. & F. R. (\%) \\
\hline Fluorosis & 17 & 54.83 \\
\hline Hipoplasia & 9 & 29.03 \\
\hline Opacidad & 3 & 9.67 \\
\hline Amelogénesis imperfecta & 1 & 3.22 \\
\hline No sabe & 1 & 3.22 \\
\hline Total & 31 & 100 \\
\hline
\end{tabular}

F. A. frecuencia absoluta $\quad$ F. $R$. frecuencia relativa

Tabla 1. Identificación de la fluorosis como defecto de esmalte. 


\begin{tabular}{|l|c|c|}
\hline & F. A. & F. R. (\%) \\
\hline Pigmentación & 16 & 51.61 \\
\hline Hipocalcificación & 4 & 12.90 \\
\hline Hipoplasia & 3 & 9.67 \\
\hline Hipomineralización & 1 & 3.22 \\
\hline Opacidad demarcada & 1 & 3.22 \\
\hline No sabe & 6 & 19.35 \\
\hline Total & 31 & 100 \\
\hline
\end{tabular}

F. A. frecuencia absoluta F. R. frecuencia relativa

Tabla 2. Identificación de Pigmentación.

29.03\% que lo confundió con una hipoplasia de esmalte y otro porcentaje mucho menor que lo identificaron como opacidad $(9.67 \%)$, y amelogénesis imperfecta (3.22\%). Solo uno de los encuestados contestó no saber qué defecto estaba en la imagen (3.22\%).

En la Tabla No. 2 puede observarse que después de ver la segunda imagen encuesta (Figura 2), para evaluar el conocimiento que tenían los odontopediatras de DDE, se encontró que 51,61\% de los encuestados sabían el defecto de esmalte que estaban observando, específicamente Pigmentación. Hubo un $12.90 \%$ que lo identificó como una hipocal-

\begin{tabular}{|c|c|c|}
\hline & F. A. & F. R. (\%) \\
\hline $\begin{array}{c}\text { Amelogénesis im- } \\
\text { perfecta }\end{array}$ & 13 & 41.93 \\
\hline Hipoplasia & 9 & 29.03 \\
\hline $\begin{array}{c}\text { Hipomineralización } \\
\text { Molar Incisivo }\end{array}$ & 5 & 16.12 \\
\hline $\begin{array}{c}\text { Dentinogénesis } \\
\text { imperfecta }\end{array}$ & 1 & 3.22 \\
\hline Hipocalcificación & 1 & 3.22 \\
\hline No sabe & 2 & 6.45 \\
\hline Total & 31 & 100 \\
\hline
\end{tabular}

F. A. frecuencia absoluta F. R. frecuencia relativa

Tabla 3. Identificación de Amelogénesis imperfecta.

\begin{tabular}{|c|c|c|}
\hline Microabrasión & 28 & $90.32 \%$ \\
\hline Blanqueamiento & 18 & $58.06 \%$ \\
\hline Carillas & 17 & $54.83 \%$ \\
\hline Resinas & 16 & $51.61 \%$ \\
\hline Coronas & 11 & $35.48 \%$ \\
\hline Flúor & 10 & $32.25 \%$ \\
\hline Sellantesw & 5 & $16.12 \%$ \\
\hline Vidrios Ionoméricos & 3 & $9.67 \%$ \\
\hline Megabrasión & 2 & $6.45 \%$ \\
\hline Profilaxis & 1 & $3.22 \%$ \\
\hline
\end{tabular}

Tabla 4. Nivel de conocimiento de tratamientos disponibles para Defectos de Esmalte.

cificación, un $9.67 \%$ como una hipoplasia y otro porcentaje mucho menor que lo identificaron como hipomineralización (3.22\%), y opacidad demarcada (3.22\%). Un 19.35\% de los encuestados contestó no saber qué defecto estaba en la imagen.

En la tercera imagen (Figura 3), se les pidió a los odontopediatras que evaluaran el defecto de esmalte: amelogénesis imperfecta. De los encuestados $41.93 \%$ acertaron con el diagnóstico. Un $29.03 \%$ lo identificó como hipoplasia de esmalte y un $16.12 \%$ como Hipomineralización Molar Incisivo. Solo un 3.22\% lo relacionaron con dentinogénesis imperfecta o hipocalcificación y por último un $6.45 \%$ no contestaron no saber cuál era el defecto de esmalte.

Por último, en la tabla No. 4 se puede observar los resultados de haber preguntado a los encuestados, que tratamientos estéticos y cosméticos conocían como alternativas para mejorar los defectos de esmalte. Un alto porcentaje $(90.32 \%)$, contestó conocer la microabrasión, un $58.06 \%$ blanqueamiento, un $54.83 \%$ carillas y $51.61 \%$ resinas. Los demás tratamientos conocidos fueron coronas $35.48 \%$, flúor $32.25 \%$, sellantes $16.12 \%$, vidrios ionoméricos $9.67 \%$, megabrasión $6.45 \%$, profilaxis $3.22 \%$. 


\section{Discusión}

De los resultados obtenidos, puede observarse que los odontopediatras tienen un adecuado conocimiento de los DDE, en especial de la fluorosis, la cual en su mayoría lograron identificar. Sin embargo, llama la atención entre los resultados, la confusión que existe sobre todo entre pigmentación e hipoplasia o Hipomineralización Molar Incisivo (HMI). Es importante destacar que la pigmentación es una coloración extrínseca o intrínseca que posee el diente, mientras que la hipoplasia es un defecto cuantitativo, representado por una falta de sustancia de esmalte en forma de fosa o fisura ${ }^{28,38}$ y la HMI es una translucencia u opacidad del esmalte, bien definida y no difusa como la fluorosis. A pesar de no ser siempre el caso, este esmalte hipomineralizado puede caerse y dejar la dentina expuesta, involucrando los primeros molares, incisivos superiores e inferiores permanentes. ${ }^{49}$

Actualmente existe una creciente demanda de tratamientos dentales estéticos en la población adolescente e infantil, que incluyen microabrasión, blanqueamientos, carillas, coronas. Existen tratamientos apropiados que maximizan la preservación de la estructura dental y evitan daños que pueden ocurrir durante la recuperación de la superficie dental. ${ }^{40}$ Sin embargo, existen casos donde la mircroabrasión no puede solucionar problemas mayores de pigmentación o pérdida de estructura, y se hacen imperativos otras alternativas terapéuticas. ${ }^{41}$

Cuando estas técnicas conservadoras han fallado para lograr el resultado deseado, el camuflaje de la opacidad con la resina compuesta puede ser útil. Nuevas técnicas tales como la infiltración o sellar la opacidad, pueden alterar el índice de refracción del esmalte, ofreciendo más opciones de tratamiento. ${ }^{42}$
No menos importante es destacar la seguridad, la eficacia, la estabilidad del color a largo plazo y el malestar (sensibilidad dental e irritación gingival), de los productos disponibles en el mercado para blanqueamiento. Es necesario que los mismos sean evaluados ${ }^{43}$ para conocer el margen de seguridad de su uso, sobre todo dentro del paciente pediátrico.

Por los resultados reportados en esta encuesta, se puede plantear que los odontopediatras conocen los tratamientos actualizados en la terapia para mejorar los defectos de esmalte. Sin embargo, éstos no deben dejar de alertar sobre la prevención de fluorosis y otros DDE, así como la importancia del adecuado diagnóstico, y explicar las opciones de tratamiento disponibles para pacientes pediátricos. ${ }^{44}$ Ya que existe desinformación si los mismos son seguros o no.

Además de todo lo anteriormente expuesto, los DDE tienen un impacto en el desarrollo de la caries dental. Ellos representan un factor de esta enfermedad multifactorial. Si se aumentan los cuidados pre y postnatales así como la nutrición de los niños, es muy probable que disminuya la aparición de caries asociada a defectos de esmalte. El control de la caries y tratamiento a molares severamente afectados es un reto para el clínico, de allí la importancia de conocer y prevenir los defectos de esmalte. Solo cuando se conozcan los factores causales de los defectos de esmalte, será posible la prevención. ${ }^{45}$

Pocos son los estudios que miden el nivel de conocimiento de los defectos de esmalte, ${ }^{46-49}$ pareciera que se le da mayor importancia en este ámbito a los relacionados con caries dental. . $^{50,51}$ Sin embargo, los DDE afectan no solo la integridad de la estructura dentaria, sino también 
acarrean problemas de sensibilidad, estética, función y además problemas de autoestima.

Es oportuno señalar que ante la presencia de DDE de esmalte como la amelogénesis imperfecta, es indicado realizar interconsultas debido a su relación con problemas renales. ${ }^{52-55}$

A pesar de este estudio contar con una reducida muestra puede ser pionero en la información acerca del nivel de conocimiento entre especialistas de DDE. Se necesitan más investigaciones para destacar la importancia de conocer y transmitir entre los odontólogos lo que son los DDE y sus consecuencias. Se hace nece- sario mejorar la difusión de técnicas simples al alcance de la población infantil que contribuirán a mejorar su percepción como individuos.

\section{Conclusión}

Los odontopediatras consultados en el presente estudio mostraron un nivel aceptable de conocimiento hacia los defectos de esmalte y los tratamientos disponibles para mejorar la apariencia de los mismos. Sin embargo, se sugiere continuar profundizando en el conocimiento, identificación y diagnóstico de los DDE a fin de lograr tratamientos adecuados y más longevos.

\section{Referencias bibliográficas}

1. Naranjo Sierra MC. Terminología, clasificación y medición de los defectos en el desarrollo del esmalte. Revisión de literatura. Univ Odontol 2013;32(68):33-44.

2. Simmer JP, Papagerakis P, Smith CE, Fisher DC, Rountrey AN, Zheng L, Hu JC. Regulation of dental enamel shape and hardness. J Dent Res. 2010; 89(10):1024-38.

3. Jalevik B, Klingberg G. Treatment outcomes and dental anxiety in 18-year-olds with MIH, comparisons with healthy controls - a longitudinal study. Int J Paediatr Dent. 2012; 22(2):85-91.

4. Jalevik B, Klingberg G. Dental treatment, dental fear and behaviour management problems in children with severe enamel hypomineralization of their permanent first molars. Int J Paediatr Dent. 2002; 12(1):24-32.

5. Basha S, Mohamed RN, Swamy HS. Prevalence and associated factors to developmental defects of enamel in primary and permanent dentition. Oral Health Dent Manag. 2014;13(3):588-94.

6. Vallejos-Sánchez AA, Medina-Solís CE, Casanova-Rosado JF, Maupomé G, Casanova-Rosado AJ, Minaya-Sánchez M. Defectos del esmalte, caries en dentición primaria, fuentes de fluoruro y su relación con caries en dientes permanentes. Gac Sanit. 2007; 21(3): 227-34.

7. Hoffmann RH, de Sousa Mda L, Cypriano S. Prevalence of enamel defects and the relationship to dental caries in deciduous and permanent dentition in Indaiatuba, Sao Paulo, Brazil. Cad Saude Publica. 2007;23(2):435-44.

8. Lunardelli SE, Peres MA. Prevalence and distribution of developmental enamel defects in the primary dentition of pre-school children. Braz Oral Res. 2005;19(2):144-9.

9. Acosta de Camargo MG. Defectos de Esmalte en la población infantil. Revisión Bibliográfica. Revista Odous Científica. 2010; 11(1):51-8

10. Nik-Hussein NN, Majid ZA, Mutalib KA, Abdullah F, Abang A, Wan MN. Prevalence of developmental defects of enamel among 16-year-old children in Malaysia. Annal Dent Univ Malaya.1999;6(4):11-6. 
11. Hoffman MP, Cutress TW, Tomiki S. Prevalence of developmental defects of enamel in children in the Kingdom of Tonga. N Z Dent J. 1988;84(375):7-10.

12. King NM. Developmental defects of enamel in chinese girls and boys in Hong Kong. Adv Dent Res. 1989 Sep;3(2):120-5.

13. Mackay TD, Thomson WM. Enamel defects and dental caries among Southland children. N Z Dent J. 2005 Jun;101(2):35-43.

14. Ferrini FR, Marba ST, Gaviao MB. Oral conditions in very low and extremely low birth weight children. J Dent Child (Chic). 2008;75(3):235-42.

15. Guergolette RP, Dezan CC, Frossard WT, Ferreira FB, Cerci Neto A, Fernandes KB. Prevalence of developmental defects of enamel in children and adolescents with asthma. J Bras Pneumol. 2009;35(4):295-300.

16. Ausar A, Kalayci AG. The presence and distribution of dental enamel and caries in children with celiac disease. Turk J Pediatr. 2008;50(1):45-50.

17. Psoter WJ, Reid BC, Katz RV. Malnutrition and dental caries: a review of the literature. Caries Res 2005; 39(6): 441-7. 18. Nikiforuk G, Fraser D. The etiology of enamel hypoplasia: a unifying concept. J Pediatr 1981;98:888-93.

19. Ibarra-Santana C, Ruiz-Rodríguez M, Fonseca-Leal M, Gutiérrez-Cantú FJ, Pozos-Guillén. Enamel Hypoplasia in Children with Renal Disease in Fluoridated Area. J Clin Pediatr Dent 2007;31(4):274-8.

20. Koch MJ, Buhrer R, Pioch T, Scharer K. Enamel hypoplasia of primary teeth in chronic renal failure. Pediatr Nephrol 1999; 13:68-72.

21. Oliver WJ, Owings CL, Brown WE, Shapiro BA. Hypoplasia enamel associated with nephrotic syndrome. Pediatrics $1963 ; 32: 399-406$.

22. Ford D, Seow WK, Kazoullis S, Holcombe T, Newman B. A controlled study of risk factors for enamel hypoplasia in the permanent dentition. Pediatr Dent. 2009;31(5):382-8.

23. Coffield KD Phillips C, Brady M, Roberts MW, Strauss RP, Wright JT. The psychosocial impact of developmental dental defects in people with hereditary amelogenesis imperfecta. J Am Dent Assoc. 2005; 136:620-30

24. Vargas FF, Ardenghi TM. Developmental enamel defects and their impact on child oral health-related quality of life. Braz. oral res. 2011; 25(6): 531-57.

25. Rodd HD, Abdul-Karim A, Yesudian G, O'Mahony J, Marshman Z. Seeking children's perspectives in the management of visible enamel defects. Int J Paediatr Dent. 2011;21(2):89-95.

26. Dean HT. Classification of Mottled Enamel Diagnosis. J Am Dent Assoc 1934;21:1421-6.

27. Clarkson J, O'Mullane D. A modified DDE Index for use in epidemiological studies of enamel defects. J Dent Res $1989 ; 445-50$.

28. FDI Commission on Oral Health, Research and Epidemiology: A review of developmental deffects on dental enamel index (DDE index) Int Dent J. 1992; 42:411-26.

29. Ghanim A, Elfrink M, Weerheijm K, Marino R, Manton D. A practical method for use in epidemiological studies on enamel hypomineralisation. Eur Arch Paediatr Dent. 2015;16(3):235-46.

30. Bermeo AP. Microabrasión de esmalte. Odontol Pediatr. 2009;8(1):18-21

31. Casas L, Baseggio W, Franco EB, Modelli RF. Tratamiento de la pigmentación sistémica y la fluorosis por medio de blanqueamiento asociado a microabrasion de esmate. Acta Odontol Venez. 2010. Disponible en: www. actaodontologica.com/ediciones/2010/2/art1.asp

32. Pini NIP, Sundfeld-Neto D, Aguiar FHB, Sundfeld RH, Martins LRM, Lovadino JR, Lima DA. Enamel microabrasion: An overview of clinical and scientific considerations. World J Clin Cases. 2015;3(1):34-41. 
33. Nashan FP, da Silva LM, Baseggio W, Franco EB, Francisconi PA, Modelli RD, Wang L. Consevative approach for a clinical resolution of enamel white spot lesions. Quintessence Int. 2011;42:423-6.

34. Sundfeld RH, Sundfeld-Neto D, Machado LS, Franco LM, Fagundes TC, Briso AL. Microabrasion in tooth enamel discoloration defects: three cases with long-term follow-ups. J Appl Oral Sci. 2014; 22: 347-54.

35. Natera A, Da Silva A, Fernández M, Montilla MA, Moukel J, Rodríguez L, Vivas S. Tratamiento de fluorosis con microabrasión del esmalte. Reporte de un caso clínico índice TF5. Odous Cientif. 2015; 16(2):51-7.

36. Amarlal D, Rayen R, Muthu MS. Macroabrasion in pediatric dentistry. J Clin Pediatr Dent. 2006;31(1):9-13.

37. Chu FC. Clinical considerations in managing severe tooth discoloration with porcelain veneer. J Am Dent Assoc. 2009;140(4):442-6.

38. Suckling GW. Developmental defects of enamel-historical and present day perspectives of their pathogenesis. Adv Dent Res 1989, 3:87-94.

39. Weerheijm KL, Jälevik B, Alaluusua S. Molar-incisor hypomineralisation. Caries Res 2001; 35(5):390-1

40. Natera AE, Peraza I, Uzcátegui G. Microabración del esmalte, técnica para la remoción de manchas dentales. Acta Odontol Venez. 2005; 43(3): 318-22.

41. Khandelwal V, Nayak UA, Nayak PA, Ninawe N. Aesthetic management of dental fluorosis. BMJ Case Rep. 2013. Disponible en: https://www.ncbi.nlm.nih.gov/pmc/articles/PMC3670045/

42. Wallace A, Deery C. Management of Opacities in children and adolescents. Dent Update. 2015;42(10):957-8

43. Pinto MM, de Godoy CH, Bortoletto CC, Olivan SR, Motta LJ, Altavista OM, Lumi K, Sobral AP, Bussadori SK.

Tooth whitening with hydrogen peroxide in adolescents: study protocol for a randomized controlled trial. Trials. 2014.

Disponible en: https://www.ncbi.nlm.nih.gov/pmc/articles/PMC4201687/pdf/13063_2014_Article_2260.pdf

44. Atia GS, May J. Dental fluorosis in the paediatric patient. Dent Update. 2013;40(10):836-9

45. Alaluusua S. Defining Developmental Enamel Defects-associated Childhood Caries: Where are we now? J Dent Res. 2012; 91(6):525-7.

46. Bonifacio SM, Moreira CR, de Oliveira FS, de Andrade MA. Tratamiento de hipoplasia de esmalte con la tecnica de microabrasion en odontopediatria. Rev Odontol Dominic. 1999; 5(1):9-14

47. Mattos-Vela Manuel Antonio, Carrasco-Loyola Milagros Bertha, Valdivia-Pacheco Suelen Giuliana. Nivel de Conocimiento sobre Pasta Dental Fluorada en Padres y Profesores de Preescolares. Int J Odontostomat. 2013; 7(1):17-24.

48. Silva MJ, Alhowaish L, Ghanim A, Manton DJ. Knowledge and attitudes regarding molar incisor hypomineralisation amongst Saudi Arabian dental practitioners and dental students. Eur Arch Paediatr Dent 2016 ;17(4):215-22.

49. Rigo L, Lodi L, Garbin RR. Differential diagnosis of dental fluorosis made by undergraduate dental students. Einstein (Sao Paulo). 2015;13(4):547-54

50. Arrieta VK, González F, Pomares AC. Conocimientos y prácticas sobre fluorosis dental en odontólogos y auxiliaries Hospital Local Cartagena de Indias. Revista Colombiana de Investigación Cientifica. 2012; 3(7):1-9 51. Contreras N, Valdivieso M, Cabello, E. Nivel de conocimientos y prácticas de medidas preventivas de profesionales de salud sobre caries dental en el infante. Rev Estomatol Herediana. 2008;18(1):29-34.

52. Quintero E, Machado R, Bisamon C, Blanchard MG, Hernandez M. Nivel de conocimiento de los padres sobre la salud bucal de escolares y su repercusión en la incidencia de caries dental. Odous Cientif. 2015;16(2):19-26

53. Acosta MG, Bolanos A, Simancas V, Landaeta A. Síndrome Amelogénesis Imperfecta-Nefrocalcinosis. Revisión bibliográfica. Acta Odontológica de Venezuela 2014;52(3).

54. Hunter L, Addy LD, Knox J, Drage N. Is amelogenesis imperfect an indication for renal examination? Int J 
Paediatr Dent 2007; 17:62-5.

55. Elizabeth J, Lakshmi Priya K, Umadevi R, Ranganathan K. Amelogenesis imperfecta with renal disease-a report of two cases. J Oral Pathol Med 2007;36:625-8.

Recibido: 13/09/2016

Aceptado 04/11/2016

Correspondencia: María Gabriela Camargo. Urb Manongo Calle La Colina 2 Res Villas de Bugambilia. Valencia. Tlf 0241894798804144287424 gabrieladecamargo@yahoo.com 